\title{
Gravitational waves as a probe of globular cluster formation and evolution
}

\author{
Isobel M. Romero-Shaw ${ }^{\circledR}$, ${ }^{\text {, }}$ Kyle Kremer, ${ }^{3,4}$ Paul D. Lasky, ${ }^{1,2}$ Eric Thrane ${ }^{1,2}$ and Johan Samsing ${ }^{\circledR 5}$ \\ ${ }^{1}$ Monash Centre for Astrophysics, School of Physics and Astronomy, Monash University, Clayton, VIC 3800, Australia \\ ${ }^{2}$ OzGrav: The ARC Centre of Excellence for Gravitational-wave Discovery, Clayton, VIC 3800, Australia \\ ${ }^{3}$ TAPIR, California Institute of Technology, Pasadena, CA 91125, USA \\ ${ }^{4}$ The Observatories of the Carnegie Institution for Science, Pasadena, CA 91101, USA \\ ${ }^{5}$ Niels Bohr International Academy, The Niels Bohr Institute, Blegdamsvej 17, DK-2100 Copenhagen, Denmark
}

Accepted 2021 June 23. Received 2021 June 15; in original form 2020 November 30

\begin{abstract}
Globular clusters are considered to be likely breeding grounds for compact binary mergers. In this paper, we demonstrate how the gravitational-wave signals produced by compact object mergers can act as tracers of globular cluster formation and evolution. Globular cluster formation is a long-standing mystery in astrophysics, with multiple competing theories describing when and how globular clusters formed. The limited sensitivity of electromagnetic telescopes inhibits our ability to directly observe globular cluster formation. However, with future audio-band detectors sensitive out to redshifts of $z \approx 50$ for GW150914-like signals, gravitational-wave astronomy will enable us to probe the Universe when the first globular clusters formed. We simulate a population of binary black hole mergers from theoretically motivated globular cluster formation models, and construct redshift measurements consistent with the predicted accuracy of third-generation detectors. We show that we can locate the peak time of a cluster formation epoch during reionization to within $0.05 \mathrm{Gyr}$ after $1 \mathrm{yr}$ of observations. The peak of a formation epoch that coincides with the Universal star formation rate can be measured to within 0.4-10.5 Gyr after $1 \mathrm{yr}$ of observations, depending on the relative weighting of the model components.
\end{abstract}

Key words: gravitational waves - stars: black holes - globular clusters: general-galaxies: star formation - dark ages, reionization, first stars - black hole mergers.

\section{INTRODUCTION}

The first detections of gravitational waves, made over the last five years (Abbott et al. 2019; Abbott et al. 2020c), provide a new lens through which to observe the Universe. Advanced LIGO (Aasi et al. 2015) and Virgo (Acernese et al. 2015) have confirmed the existence of multiple phenomena that, prior to the era of gravitational-wave astronomy, had only been theoretically proposed; stellar mass binary black holes (BBH; Abbott et al. 2016), merging neutron stars (Abbott et al. 2017b,c), and intermediate-mass black holes (Abbott et al. 2020b) have all been directly observed with gravitational waves.

We are accruing gravitational-wave observations of merging black holes at an accelerating rate (Abbott et al. 2016, 2019, 2020c,d). This abundance of BBH merger detections presents a variety of puzzles across theoretical astrophysics. One such question is how BBH systems that merge within the age of the Universe are assembled. If the binary evolves in isolation, this outcome may be achieved via the common envelope process (see e.g. Livio \& Soker 1988; Bethe \& Brown 1998; Ivanova et al. 2013; Kruckow et al. 2016), stable mass transfer of a stellar secondary on to the primary black hole (van den Heuvel, Portegies Zwart \& de Mink 2017; Neijssel et al. 2019; Bavera et al. 2021), chemically homogeneous evolution (de Mink et al. 2010; de Mink \& Mandel 2016) and/or ambient gas-driven fallback (Tagawa, Kocsis \&

^E-mail: isobel.romero-shaw@monash.edu
Saitoh 2018). Alternatively, the compact object binary may form dynamically. In this case, the two components evolve separately, only encountering one another once they are already black holes. For this encounter to take place, the components must reside in an environment facilitating dynamical interactions. Such environments include active galactic nuclei (e.g. Gröbner et al. 2020), nuclear star clusters (e.g. Fragione \& Kocsis 2018; Hoang et al. 2018), young massive clusters (Di Carlo et al. 2019), and globular clusters (e.g. Rodriguez et al. 2015; Hong et al. 2018). In globular clusters, mass segregation leads to the formation of a dark compact-object core (see e.g. Morscher et al. 2015; Wang et al. 2016; Kremer et al. 2020a), where black holes may interact and merge dynamically. Evidence from LIGO-Virgo's third observing run suggests that a substantial fraction $(\approx 25-93$ per cent with 90 per cent credibility) of merging BBH form dynamically (Abbott et al. 2020d); see also Abbott et al. (2020a,b), Romero-Shaw et al. (2020b), and Zevin et al. (2021).

The gravitational-wave signal from a binary compact object merger carries information about the source's component masses, component spins, and orbital eccentricity. These parameters can be used to distinguish which formation channel the binary evolved through. When a BBH system evolves in isolation, it is expected to have component masses $m \lesssim 65 \mathrm{M}_{\odot}$ due to the effects of pairinstability supernovae (e.g. Heger \& Woosley 2002; Fishbach \& Holz 2017; Talbot \& Thrane 2018; see Belczynski 2020 for a review of recent updates to this limit for various stellar populations). The coevolution of the binary is thought to lead to component spins that are 
preferentially aligned with the orbital angular momentum (Kalogera 2000; Campanelli, Lousto \& Zlochower 2006; Stevenson, Berry \& Mandel 2017; Talbot \& Thrane 2017), and since compact binary orbits circularize through gravitational radiation at a faster rate than their separation reduces, any orbital eccentricity induced by the supernovae of the components becomes negligible by the time the gravitational-wave signal enters the observing band (Peters 1964; Hinder et al. 2008). ${ }^{1}$ When a BBH system forms and merges dynamically, its properties can be detectably different from those of isolated mergers. In the dense environments that support dynamical formation, repeated $\mathrm{BH}$ or stellar mergers can give rise to binaries in which one or both components have masses within the pulsational pair-instability mass gap (e.g. Gerosa \& Berti 2017; Samsing \& Hotokezaka 2020; Kimball et al. 2020b; Kremer et al. 2020b). Because the components do not co-evolve, their spins may have any orientation relative to each other (Rodriguez et al. 2016b), and few-body interactions and/or gravitational-wave captures can give rise to mergers with non-negligible eccentricity close to merger (see e.g. Samsing, MacLeod \& Ramirez-Ruiz 2014; Rodriguez et al. 2018c; Gondán \& Kocsis 2019; Samsing et al. 2018; Zevin et al. 2019; Kremer et al. 2020a). In globular clusters, $\sim 5$ per cent of all $\mathrm{BBH}$ mergers are expected to have significant eccentricity close to merger ( $e \geq 0.1$ at $10 \mathrm{~Hz}$; Samsing 2018; Samsing \& D'Orazio 2018; Rodriguez et al. 2018a,c).

Globular clusters are observed in great quantities, both inside our Galaxy and beyond; there are $\approx 160$ known globular clusters in the Milky Way (SEDS Messier Data base 2020), $\approx 500$ in the neighbouring Andromeda galaxy (Peacock et al. 2010), and $\sim 12000$ in supergiant elliptical galaxies like M87 (Tamura et al. 2006). Despite their prolific nature, it is not known how globular clusters form. Globular clusters contain stars that are thought to be among some of the most ancient in their host galaxy (for example, the globular cluster Hpl contains some of the most ancient stars ( $\gtrsim 12$ Gyr) in the Milky Way; Kerber et al. 2019), making their formation difficult to observe with electromagnetic telescopes. To date, the primary method to constrain cluster ages is main-sequence fitting of colour-magnitude diagrams (e.g. Gratton et al. 1997; Sarajedini et al. 2007; VandenBerg et al. 2013) with a small subset of cluster ages also determined from the white dwarf cooling sequence (e.g. Hansen et al. 2013; GarcíaBerro et al. 2014). Typical globular cluster age measurements have uncertainties of order $\mathcal{O}(1 \mathrm{Gyr})$; see Forbes et al. $(2015,2018)$ and references therein. It is hoped that the James Webb Space Telescope (JWST) - due to be launched in 2021 October (NASA 2020) - will be able to constrain cluster ages to within $1 \mathrm{Gyr}$ (e.g. Correnti et al. 2016).

Measurements of globular cluster ages and metallicities suggest two different globular cluster sub-populations: very old globular clusters, which are observed to have a wide range of ages and metallicities; and younger globular clusters, which have metallicities that anticorrelate with their ages (Forbes \& Bridges 2010; Hansen et al. 2013; Leaman, VandenBerg \& Mendel 2013; VandenBerg et al. 2013; Forbes et al. 2015). Current theories of globular cluster formation fall into two main categories: (i) clusters formed as a byproduct of active star formation in galaxy discs (e.g. Elmegreen 2010; Shapiro, Genzel \& F'orster Schreiber 2010; Kruijssen 2015)

\footnotetext{
${ }^{1}$ While Kozai-Lidov resonance (Kozai 1962; Lidov 1962) is predicted to lead to eccentric mergers and misaligned spins, the Kozai-Lidov field merger rate is thought to be small (e.g. Antonini, Toonen \& Hamers 2017; Fishbach, Holz \& Farr 2017; Silsbee \& Tremaine 2017; Rodriguez \& Antonini 2018; Fragione \& Kocsis 2019).
}

and (ii) clusters formed due to the collapse of dark matter haloes during or before the epoch of reionization (e.g. Fall \& Rees 1985; Katz \& Ricotti 2014; Ramirez-Ruiz et al. 2015; Trenti, Padoan \& Jimenez 2015; Kimm et al. 2016; Ma et al. 2021). In category (i), the formation probability follows the observed star formation rate (SFR; Madau \& Dickinson 2014), peaking at $z \approx 2.5$ (Forbes et al. 2015, 2018), while in category (ii) the formation probability peaks at $6 \lesssim$ $z \lesssim 12$ (Forbes et al. 2015; Trenti et al. 2015).

Constraining the primary formation epoch of globular clusters will answer long-established questions in astrophysics. If globular clusters predominantly form before $z \approx 6$, they may play a leading role in the reionization of the Universe (e.g. Ma et al. 2021). On the other hand, if the globular cluster formation probability curve follows the SFR, and the majority of star formation takes place in such environments (e.g. Lada \& Lada 2003), then detailed understanding of cluster formation histories may place critical constraints upon the overall SFR. If we know the formation epoch of clusters, then we can adjust N-body simulations to more correctly reproduce clusters observed at $z=0$, thereby enhancing our physical descriptions of cluster initial conditions. Our understanding of the role that globular clusters play in the evolution of galaxies - for example, whether globular clusters are early galaxies (Elmegreen \& Elmegreen 2017), failed galaxies (Fall \& Rees 1985), or galaxy remnants (Majewski et al. 2000) - can also be improved by observing globular clusters as they form and evolve.

As detectors improve, gravitational waves will allow us to trace compact binary mergers throughout cosmic time (see e.g. Vitale et al. 2019; Safarzadeh et al. 2019). In turn, this will allow us to use gravitational waves as probes of cluster formation and evolution. The current generation of detectors can observe events out to redshifts $z \lesssim 1.5$ - not far enough for globular cluster formation to be traced through our observations. In order to examine globular cluster formation, we must wait for third-generation gravitational-wave observatories such as the Einstein Telescope (Punturo et al. 2010) and Cosmic Explorer (Abbott et al. 2017a). These observatories, proposed to begin taking data ca. 2035, will be able to detect $\mathrm{BBH}$ mergers with total mass of order $\mathcal{O}(100) \mathrm{M}_{\odot}$ out to redshifts $z \approx 30$, and GW150914-like mergers out to $z \approx 50$ (Hall \& Evans 2019).

In this paper, we demonstrate the power of gravitational-wave observations as probes of globular cluster formation and evolution. In Section 2.1, we motivate a Gaussian mixture model describing the globular cluster formation probability over cosmic time. We explain the metallicity-dependent merger time distribution used to convert this underlying globular cluster formation probability to the BBH merger probability in Section 2.2. In Section 3, we outline our population inference method. We test our ability to recover the underlying globular cluster formation probability in Section 4 , obtaining population inference results using simulated third-generation gravitational-wave observatory data with realistic uncertainties. For these simulations, we use only mergers that are massive and rapidly merging - signatures of dynamical formation as 'snapshots' of the clusters at creation. We find that we can measure the formation epochs of globular clusters to $0.02-0.6$ Gyr precision at 99 per cent confidence after $1 \mathrm{yr}$ of third-generation gravitationalwave observations - comparable to the forecasted accuracy of JWST (e.g. Correnti et al. 2016) - unless cluster formation primarily occurs during reionization, in which case the precision with which we can locate a secondary lower redshift formation epoch is reduced to $\mathcal{O}(10)$ Gyr. If we use all cluster mergers instead of just the small fraction that we consider to be identifiable as such, our constraints on cluster formation epochs tighten by up to an order of magnitude. 
Table 1. Parameter values chosen for our fiducial globular cluster formation probability models, used in the injection studies described in Section 4. Each of the two Gaussian peaks in our model has mean $\mu_{j}$ and standard deviation $\sigma_{j}$, where $j=1$ refers to the SFR-driven peak and $j=2$ refers to the reionization-driven peak. The ratio $w_{2} / w_{1}$ determines the relative weight of the reionization-driven peak against the SFR-driven peak. We vary $w_{2} / w_{1}$ to test our ability to recover the underlying globular cluster formation probability in three different scenarios, between which the dominant formation mechanism of clusters varies.

\begin{tabular}{lccc}
\hline & \multicolumn{3}{c}{ Value } \\
\hline Parameter & $z$ & & $t(\mathrm{Gyr})$ \\
$\mu_{1}$ & 2.00 & & 3.30 \\
$\sigma_{1}$ & 1.50 & & 2.32 \\
$\mu_{2}$ & 10.00 & & 0.48 \\
$\sigma_{2}$ & 1.35 & & 0.09 \\
$w_{2} / w_{1}$ & & $1 / 9, \quad 1, \quad 9$ & \\
\hline
\end{tabular}

In Section 5, we state the assumptions and caveats underlying our model. We conclude in Section 6.

\section{MODEL}

In the following section, we describe our model, which combines theoretically motivated globular cluster formation probability distributions (described in Section 2.1) with simulated BBH merger distributions (described in Sections 2.2 and 2.3). We assume a flat $\Lambda$ CDM Universe with $H_{0}=67.7 \mathrm{kms}^{-1} \mathrm{Mpc}^{-1}$ and $\Omega_{0}=$ 0.307 (Planck Collaboration XIII 2016).

\subsection{Globular cluster formation probability distribution}

Our globular cluster formation probability is modelled by a twocomponent Gaussian mixture model in redshift. The first component represents SFR-driven globular cluster formation, with mean $\mu_{1}$, standard deviation $\sigma_{1}$ and weight $w_{1}$; the other represents reionization-driven globular cluster formation, with mean $\mu_{2}$, standard deviation $\sigma_{2}$ and weight $w_{2}$.

We simulate a fiducial globular cluster formation probability using specific parameter values shown in Table 1. For the injection sets, we set the mean and standard deviation of the SFR-driven peak in order to best represent the true shape of the SFR (Madau \& Dickinson 2014). The mean of the reionization-driven peak is motivated by the results of Trenti et al. (2015); see also Ramirez-Ruiz et al. (2015).

To investigate our ability to distinguish the preferred channel of globular cluster formation, we vary the weight ratio $w_{2} / w_{1}$. We consider three cases: (i) globular clusters are formed primarily as a byproduct of the SFR, and there is a small contribution formed during reionization $\left(w_{2} / w_{1}=1 / 9\right)$; (ii) globular clusters are formed with equal probability during reionization and through star formation $\left(w_{2} / w_{1}=1\right)$; and (iii) globular clusters are formed primarily during reionization, with a small contribution forming in accordance with the SFR $\left(w_{2} / w_{1}=9\right)$. All three cases lead to a similar merger probability at $z=0$, so the scenarios cannot be distinguished by existing detectors. However, third-generation detectors will be able to constrain $w_{2} / w_{1}$. Our three globular cluster formation probability functions are plotted in pink in the three panels of Fig. 1.

\subsection{Binary black hole merger probability distribution}

In order to translate globular cluster formation probability into gravitational-wave observables, we calculate the distribution of BBH mergers in globular clusters. To simulate globular cluster evolution, we use the CMC Cluster Catalog (Kremer et al. 2020a). These simulations were computed using CMC (Joshi et al. 2000; Pattabiraman et al. 2013), a Hénon-type Monte Carlo code that includes various physical processes relevant to the dynamical formation of $\mathrm{BH}$ binaries including two-body relaxation, stellar and binary evolution (computed using updated versions of SSE and BSE; Hurley, Pols \& Tout 2000; Hurley, Tout \& Pols 2002), and direct integration of small- $N$ resonate encounters (Fregeau \& Rasio 2007) including post-Newtonian effects (Rodriguez et al. 2018b). A number of parameters relevant to the long-term cluster evolution are varied within this set of simulations (namely the total cluster mass, initial virial radius, metallicity, and radial position within the Galactic potential), with values chosen to reflect the observed properties of the Milky Way globular clusters. Altogether, this catalogue nearly completely covers the full parameter space of the Milky Way globular clusters and captures the formation of a variety of astrophysical objects such as gravitational-wave sources as well as X-ray binaries, pulsars, and blue stragglers. By implementing a

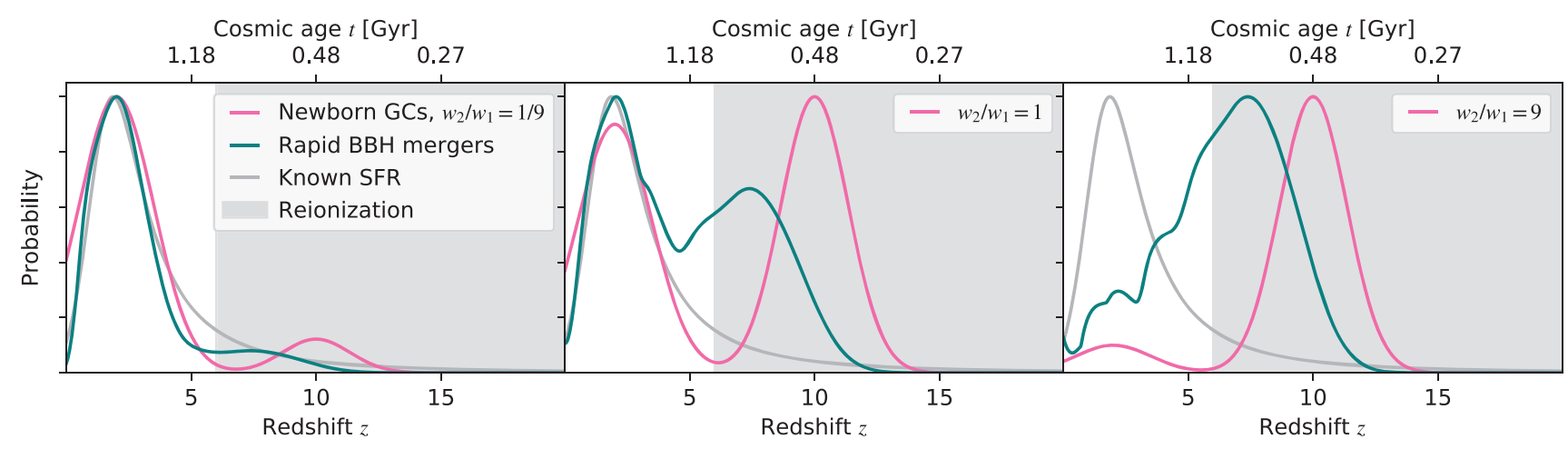

Figure 1. The three cases we consider in this work are illustrated from left to right: $w_{2} / w_{1}=1 / 9$, where SFR-driven globular cluster formation dominates clusters formed by $z=0 ; w_{2} / w_{1}=1$, where there is equal contribution to clusters formed by $z=0$ from both formation channels; and $w_{2} / w_{1}=9$, where reionization-driven globular cluster formation dominates clusters formed by $z=0$. The probability distributions of rapid-merging first-generation massive $\mathrm{BBH}$ mergers (where both components have $30 \mathrm{M}_{\odot} \leq m \leq 40 \mathrm{M}_{\odot}$ ) are plotted in teal, while the formation probability distributions of GCs in each model are plotted in pink. 


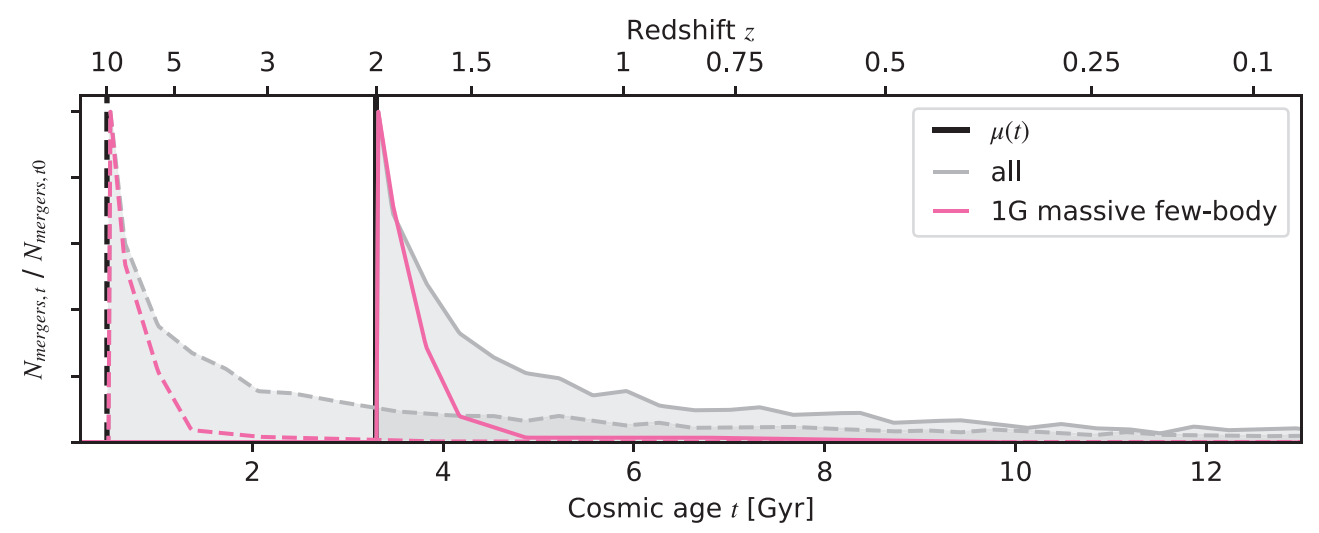

Figure 2. Merger time distributions for BBH mergers from two clusters formed at $\mu_{1}\left(z=10\right.$; during the peak of the SFR) and $\mu_{2}$ ( $z=2$; during reionization), with distributions shown with the solid and dashed curves, respectively. The distributions of all mergers from each cluster after its formation are shown in grey. The distributions of just the massive first-generation $\left(35 \mathrm{M}_{\odot} \leq m \leq 40.5 \mathrm{M}_{\odot} ; 1 \mathrm{G}\right)$ mergers formed through gravitational-wave capture during few-body interactions are shown in pink.

cluster age distribution model from El-Badry et al. (2019), Kremer et al. (2020a) estimated a BBH merger rate of roughly $20 \mathrm{Gpc}^{-3} \mathrm{yr}^{-1}$ in the local Universe, consistent with previous theoretical work on the subject (e.g. Rodriguez, Chatterjee \& Rasio 2016a; Askar et al. 2017; Rodriguez \& Loeb 2018; Antonini \& Gieles 2020) as well as with the observational rate inferred from the second LIGO/Virgo catalogue (Abbott et al. 2020c).

Each newborn globular cluster in our model has a metallicitydependent merger time distribution. The clusters in the CMC Cluster Catalog have one of three absolute metallicities: 0.0002, 0.002 , and 0.02 . We combine mergers from clusters with the same metallicity, then sort the mergers into 100 time bins. We use a univariate spline to smoothly interpolate between the bin heights. We then perform a two-dimensional linear interpolation between these smoothed merger distributions of both cluster age and metallicity. To calculate the merger distribution for a cluster born at a certain redshift, we assume for simplicity that metallicity increases linearly with the age of the Universe. We assume a metallicity of $Z=0.0002$ at a redshift of 24 and a metallicity of $Z=0.02$ at the present day (see e.g. fig. 6 of Lilly, Carollo \& Stockton 2002, for observationally driven proposals for metallicity evolution over cosmic time).

We convert the sum of merger time distributions from all clusters into a probability distribution in redshift, from which we draw our source population. In Fig. 1, the merger probability distributions from each of the three globular cluster formation probability models are plotted with teal curves. In Fig. 2, we illustrate in grey the merger time distributions for two clusters: one formed at $z=2$ and one formed at $z=10$.

\subsection{Rapid mergers as cluster formation snapshots}

The merger time for a $\mathrm{BBH}$ formed dynamically in a stellar cluster is determined by three time-scales: (i) the cluster formation time, (ii) the time required for $\mathrm{BBH}$ formation through dynamical encounters, and (iii) the gravitational-wave inspiral time from the time of last dynamical encounter to merger. The latter two timescales are sensitive to a variety of host cluster properties including total cluster mass, half-mass radius, and $\mathrm{BH}$ mass distribution (e.g. Rodriguez et al. 2016b; Askar et al. 2017; Kremer et al. 2020a). As a consequence, disentangling the cluster formation time distribution from the merger time distribution for a given list of dynamical BBH mergers may pose a challenge. This challenge may be circumvented by looking at specific classes of mergers known to have prompt merger times, $t_{\text {merge }} \lesssim \mathcal{O}(100 \mathrm{Myr})$ since cluster formation. For these rapid mergers, the observed merger time distribution much more closely traces the underlying cluster formation time distribution.

Rapid mergers in globular clusters are expected to have two primary characteristics: high eccentricities and high masses. During small- $N$ (few-body) resonant encounters, pairs of BHs can form that merge rapidly, making them more likely to retain orbital eccentricity at detection. As discussed in Samsing (2018) and Kremer et al. (2020a), roughly 5-10 per cent of cluster mergers are expected to retain high eccentricity $(e \geq 0.1)$ close to merger $\left(f_{\mathrm{GW}}=10 \mathrm{~Hz}\right)$. These binaries can have gravitational-wave inspiral times as short as days (e.g. Zevin et al. 2019); this makes them ideal tracers of cluster formation, as they merge relatively quickly after the cluster forms and are more likely to retain the dynamically induced eccentricity that can reveal their formation channel. As a natural consequence of dynamical friction, the most massive BHs in a cluster are expected, on average, to be the first to form BBHs and the first to merge (e.g. Morscher et al. 2015). Thus, BHs with masses near the assumed upper limit of the $\mathrm{BH}$ mass distribution $\left(40.5 \mathrm{M}_{\odot}\right.$ in the CMC Cluster Catalog) that merge through gravitational-wave capture encounters are ideal rapid merger candidates. For the anaysis presented in Section 4, we consider only globular cluster binaries that merge through resonant few-body encounters.

There is an inherent additional delay associated with secondgeneration $\mathrm{BHs}$ formed through previous $\mathrm{BH}$ mergers that remain bound to their host cluster. Although these will preferentially merge again quickly (within a few $10 \mathrm{Myr}$ of the previous merger) due to their relatively high mass, we do not include second-generation mergers in this analysis. Here, we consider only those massive fewbody mergers that are first-generation $(1 \mathrm{G})$, having both component masses above $35 \mathrm{M}_{\odot}$ and below $40.5 \mathrm{M}_{\odot}$. We plot this distribution of mergers in pink in Fig. 2. Over the redshift range that we study, the fraction of $1 \mathrm{G}$ massive few-body mergers varies between 3 percent and 6 per cent of all cluster mergers. We construct the merger time distribution using only $1 \mathrm{G}$ massive few-body mergers, and draw only 5 per cent of the number of detections expected from the observing durations. 


\section{METHOD}

We simulate redshift posterior probability distributions for a population of BBH mergers, and use the population inference framework to discern the injected distribution of the population. The likelihood for the data $\mathbf{d}$ is

$\mathcal{L}_{t o t}(\mathbf{d} \mid \mathbf{\Lambda})=\prod_{i}^{N} \frac{\mathcal{Z}_{\varnothing}\left(d_{i}\right)}{n_{i}} \sum_{k}^{n_{i}} \frac{\pi\left(\theta_{i}^{k} \mid \boldsymbol{\Lambda}\right)}{\pi\left(\theta_{i}^{k} \mid \varnothing\right)}$.

In this equation, $\Lambda$ is the set of parameters describing the population distribution, while $\theta_{i}^{k}$ are the parameters describing the $k$ th posterior sample of event $i$ (in our case, $\theta_{i}$ is only one parameter - redshift). Each event $i$ has $n_{i}$ posterior samples; there are a total of $N$ events. The sampling prior used for inference on data $d_{i}$ for event $i$ is $\pi\left(\theta_{i} \mid \varnothing\right)$, which is reweighted to obtain results for a population-based prior $\pi\left(\theta_{i} \mid \Lambda\right)$. The evidence obtained with the original sampling is $\mathcal{Z}_{\varnothing}\left(d_{i}\right)$. In our case, the population prior $\pi\left(\theta_{i} \mid \Lambda\right)$ is the distribution described in Section 2.

Distance measurement uncertainties are likely to be $\mathcal{O}$ (10 per cent) for most binaries observed with third-generation detectors (Vitale \& Evans 2017; Zhao \& Wen 2018). To model uncertainty of approximately this magnitude, we assume Gaussian likelihoods of width $\sigma_{z_{i}}=0.1 z_{i}$. These likelihoods each have a mean $\mu_{z_{i}}=z_{i}+r_{i}$, where $r_{i}$ is a random offset drawn from a Gaussian of mean $\mu_{r_{i}}=0$ and $\sigma_{r_{i}}=\sigma_{z_{i}}$. We produce a posterior curve by multiplying the likelihood by a uniform sampling prior. (This prior is divided out in the calculation of equation 1.) From this posterior curve, we draw 50 simulated posterior samples for each event.

To execute our population analysis, we use the Bayesian inference library bi lby (Ashton et al. 2019; Romero-Shaw et al. 2020a). We use uniform priors over all parameters. The prior covers the range $10^{-5} \leq z \leq 6$ for $\mu_{1}, 0.5 \leq z \leq 6$ for both $\sigma$ values, and $6 \leq z \leq 20$ for $\mu_{2}$. The prior on $w_{2} / w_{1}$ ranges from $10^{-2}-10$.

The formation channel of a binary may be identified using a method such as that developed in Kimball et al. (2020a), in which a BBH merger's mass and spin measurements are used to calculate its probability of being a hierarchical merger in a globular cluster. Similar methods may be extended to incorporate eccentricity measurements, which will be illuminating for globular clusters as we expect $\sim 5$ per cent of globular cluster mergers to have eccentricity $e \geq 0.1$ at $10 \mathrm{~Hz}$ (see e.g. Samsing 2018). While precession is considered a hallmark of dynamical mergers, there have been relatively few events that have clear precession measurements (Abbott et al. 2019, 2020c); however, both precession and anti-aligned spins can be measured at a population level, as demonstrated in (Abbott et al. 2020d). With third-generation detectors, component spins and precession will be well measured (e.g. Vitale \& Whittle 2018). Using such measurements at both an individual and population level, it may be possible to estimate the sub-population of globular cluster mergers within a set of $\mathrm{BBH}$ mergers from a variety of formation channels.

In this paper, we assume that mergers identified as cluster mergers have 0 per cent probability of having formed via a different channel. Such definitive statements are unlikely to be made based on the parameters of detected binaries for the vast majority of sources even if we allow for future improvements to our mechanisms for performing such identifications. In the future, binaries that form in globular clusters but are kicked out before merging may still be indistinguishable from isolated mergers, and those that do merge inside the cluster are likely to have properties similar to those in other dynamical environments (e.g. AGN discs and galactic nuclei) or field triples undergoing Kozai-Lidov resonance. More complex future analyses should weight the samples from each event by the probability that each binary formed inside a globular cluster. This is an additional complication that can be built upon the method presented here, and is left for future work.

\section{INJECTION STUDIES}

The Universal merger rate implied by current $\mathrm{BBH}$ merger observations is $\sim 0.2 \mathrm{~min}^{-1}$ (Abbott et al. 2018). For an all-seeing detector, this translates to a BBH signal detection count of $\mathcal{O}(500) \mathrm{d}^{-1}$ (Maggiore et al. 2020). Third-generation detectors such as Cosmic Explorer and the Einstein Telescope will be close to all-seeing, detecting GW150914-like events out to redshifts $z \approx 50$, and GW190521like out to $z \approx 30$ (Hall \& Evans 2019). We use 500, 10000 and 100000 as order-of-magnitude estimates for the total number of GC mergers in $1 \mathrm{~d}, 1 \mathrm{month}$, and $1 \mathrm{yr}$, respectively. This is a reasonable approximation if GC mergers make up $\geq 10$ per cent of all mergers in the Universe. We assume, based on the fraction of massive and quickmerging binaries observed in the cluster simulations described in Section 2 , that only 5 per cent of these observations can be confidently identified as cluster mergers. To simulate a month's worth of confidently identified cluster mergers, for example, we use 500 events.

We simulate data after $1 \mathrm{~d}, 1$ month, and $1 \mathrm{yr}$ of observing, for three different models of the underlying globular cluster formation probability. The first case we consider is one where globular clusters do not form efficiently during reionization. In this case, the globular cluster formation probability curve closely follows the observed SFR. We set the weight ratio $w_{2} / w_{1}=1 / 9$, such that 90 per cent of all clusters form within the SFR peak. In the second case, we set the weight ratio $w_{2} / w_{1}=1$, which leads to a $50-50$ split between clusters contributed from each peak. The final case we consider is one where globular clusters primarily form during reionization, such that 90 per cent of clusters are formed within the reionization peak. We set $w_{2} / w_{1}=9$. For all three injected data sets, the remaining four parameters $\left(\mu_{1}\right.$, $\mu_{2}, \sigma_{1}, \sigma_{2}$ ) are fixed to the values provided in Table 1 . We show the globular cluster formation probability curve and resulting probability distribution of observable BBH mergers for all three cases in Fig. 1.

In Fig. 3, we plot the injected underlying globular cluster formation probability curve in pink, and compare it to the recovered median and 99 per cent confidence intervals in grey. For all three observing periods, the injected distribution is within the 99 per cent confidence interval. Probability distributions on the five populations parameters $\mu_{1}, \mu_{2}, \sigma_{1}, \sigma_{2}$, and $w_{2} / w_{1}$ are provided as the corner plots in Appendix A. We state the widths of the 99 percent confidence intervals around $\mu_{1}$ and $\mu_{2}$ for each study in Table 2 .

We repeat the above injection studies for two additional scenarios. In the first, we neglect any measurement uncertainty and assume that each source is represented by a delta function at its true value of $z$. In this case, we see negligible change in the widths of the 99 per cent credible intervals around $\mu_{1}$ and $\mu_{2}$. In the second, we include redshift measurement uncertainty, but optimistically assume that all cluster mergers can be confidently identified, thereby allowing us to use 100 percent of the mergers from the CMC Cluster Catalog to construct our model. This leads to a reduction of up to an order of magnitude in the width of the 99 percent credible interval around $\mu_{1}$ and $\mu_{2}$; for $1 \mathrm{~d}$ of observing all cluster mergers, the credible intervals are nearly identical to those seen for a month of observing only $1 \mathrm{G}$ massive few-body mergers. These measurements are more precise - despite the longer average merger time-scale because we are able to use 20 times as many events to probe the cluster formation rate. The precision with which we can measure globular cluster formation epochs with third-generation observations 


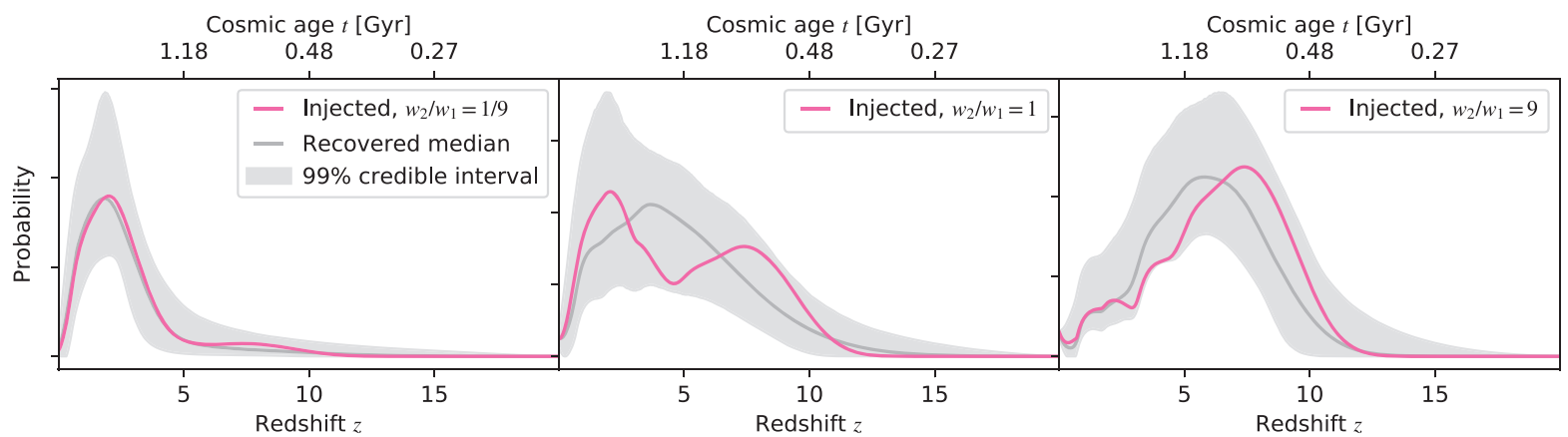

(a)

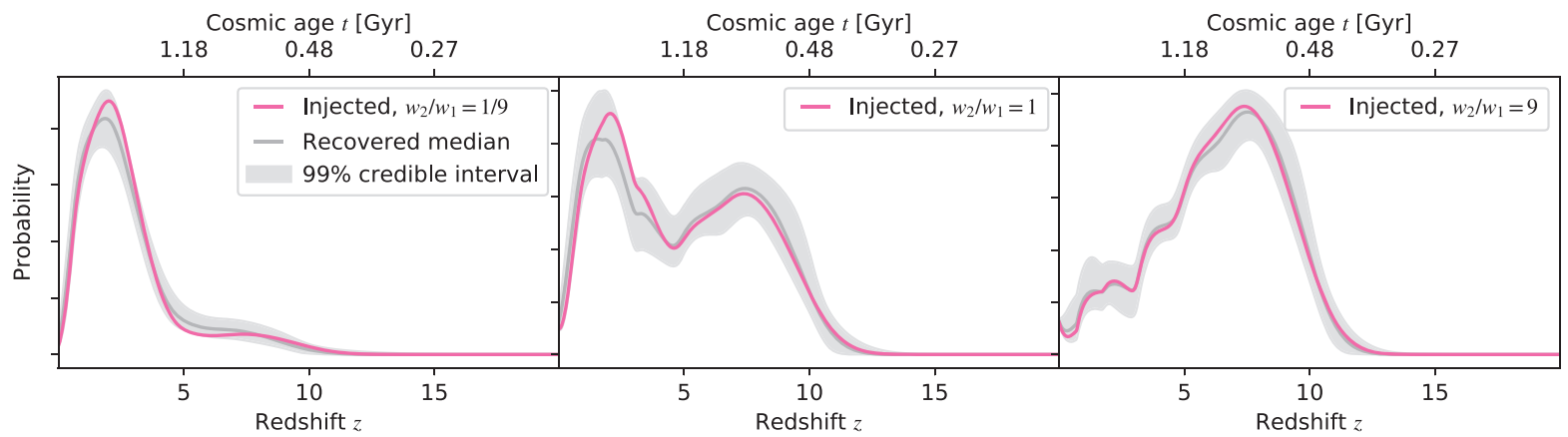

(b)

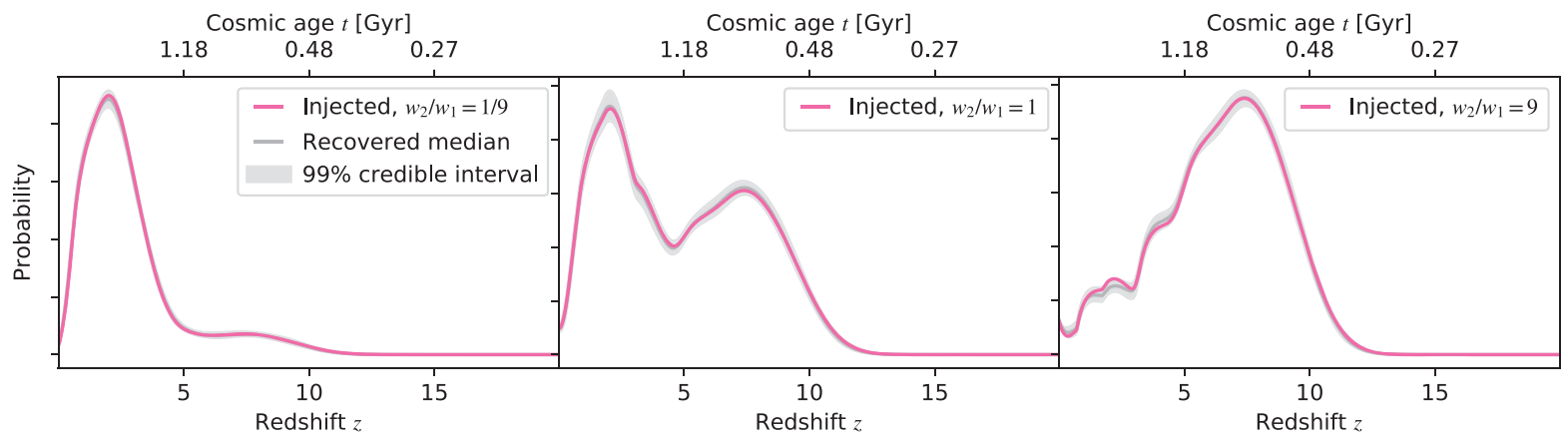

(c)

Figure 3. Globular cluster formation rates inferred from simulated third-generation detector observations of first-generation massive few-body mergers after (a) $1 \mathrm{~d}$ (using $251 \mathrm{G}$ massive few-body mergers from the total population of 500), (b) 1 month (using 500 of 10000 ), and (c) $1 \mathrm{yr}$ of detections (using 5000 of 100000). Each panel represents an underlying model with a different weighting of the two components of the Gaussian mixture model that represents the globular cluster formation rate. Left: SFR-driven peak dominates formation rate; Middle: Each peak contributes equally to formation rate; Right: Reionization-driven peak dominates formation rate.

therefore sensitively depends on the number of events that are confidently identified as globular cluster mergers.

\section{SYSTEMATIC ERROR ANALYSIS AND CAVEATS}

We make a number of simplifying assumptions and approximations in our analysis, allowing us to demonstrate a generic way to probe globular cluster formation using gravitational-wave detections. These are listed in this section, with the aim to reduce the number of assumptions we make in future work that builds upon this paper.

We approximate both epochs of globular cluster formation as simple Gaussians in redshift. However, the true SFR determining the shape of the cluster formation probability does not follow a Gaussian, and the shape of the reionization-driven cluster formation probability is not known. More complex future extensions of this work may allow the shape of the Gaussians to vary, with the skewness of the Gaussian a model variable. We assume that the redshift posterior distributions are also Gaussian, but the true shape of the uncertainty distribution would vary depending on the noise in the data containing each signal.

We set our injection studies in an optimistic future where merger channels can be perfectly distinguished. While we analyse the merger distribution of only those globular cluster BBH that are high mass, rapid merging, and highly likely to be detectably eccentric (signatures of dynamical origin) for our primary results, we still ignore any possibility of contamination from other dynamical formation channels that produce mergers with similar properties, such as mergers in AGN or Kozai-Lidov (Kozai 1962; Lidov 1962) triples in the field. 
Table 2. Width of 99 per cent credible intervals (CIs) around $\mu_{1}$ (top) and $\mu_{2}$ (bottom) for each injection study described in Section 4. For these injection studies we use only first-generation massive few-body mergers, and include a 10 per cent uncertainty on source redshift. When the 10 per cent uncertainty is removed, the width of these uncertainty intervals does not meaningfully change.

\begin{tabular}{ccccr}
\hline$\mu_{1}$ & \multicolumn{3}{c}{99 per cent CI width (Gyr) } \\
& $w_{2} / w_{1}$ & Day & Month & Year \\
\hline 9 & 11.92 & 12.01 & 10.49 \\
1 & 11.99 & 9.87 & 0.53 \\
& $1 / 9$ & 10.44 & 4.32 & 0.39 \\
$\mu_{2}$ & & & \\
& & & & 0.02 \\
& 9 & 0.48 & 0.06 & 0.02 \\
& 1 & 0.74 & 0.42 & 0.05 \\
\hline
\end{tabular}

We also do not account for any sources redshifting out of band due to high masses or high eccentricities at high redshift. We do not account for the disruption/creation of globular clusters during galaxy mergers; while the increased star formation of merging galaxies should be absorbed into the SFR peak of our models, the shape of the merger time distribution at a given epoch will differ if clusters are disrupted/created at that time due to galaxy mergers, even if the overall number of globular clusters remains the same.

We assume that metallicity increases linearly with the age of the Universe to obtain different merger time distributions for clusters born at different times, but do not consider a time-evolving initial mass/density function. Metallicity, mass, and density may become globular cluster variables in future iterations of our model, allowing us to consider whether the physical properties of clusters can be inferred from observations of gravitational waves.

\section{CONCLUSIONS}

In this work, we show that observations from third-generation gravitational-wave detectors will allow us to measure the formation epochs of the population of globular clusters. Our primary results are obtained assuming that only first-generation massive few-body mergers can be confidently identified as globular cluster mergers. If up to 50 percent of clusters are born following the SFR, a cluster formation epoch at $z=2$ can be resolved to within less than 1 Gyr precision after $1 \mathrm{yr}$ of observing; however, if the majority of clusters are born during reionization, the time of this epoch will have an uncertainty $\mathcal{O}(10)$ Gyr. For all scenarios, a cluster formation epoch at $z=10$ can be resolved to within less than 1 Gyr precision after just $1 \mathrm{~d}$ of observing, and to within $0.05 \mathrm{Gyr}$ after a full year. If we are able to confidently identify all globular cluster mergers as such, these uncertainty bands can decrease by up to an order of magnitude. With third-generation detectors Cosmic Explorer and Einstein Telescope due to commence observing in the 2030s (Reitze et al. 2019; Maggiore et al. 2020), the question of how globular clusters formed may be answered with gravitationalwave observations within the next twenty years. These results will be complementary to measurements from electromagnetic telescopes, such as JWST, which will be able to constrain the ages of individual clusters to within $\mathcal{O}$ (1) Gyr (e.g. Correnti et al. 2016).

Our method can be extended for use in multiple future projects. For example, we could assign a population of mergers from multiple formation channels with a certain probability of being globular cluster mergers, and weight their contribution to the total merger distribution accordingly. We could also use intrinsic binary parameters, e.g. mass and eccentricity, to infer properties of their host clusters, like their densities at formation. The simple procedure outlined in this paper must be refined before application to real data. In particular, future work should address the fact that many signals from the more massive and highly eccentric sources may be redshifted out of band. We leave potential extensions and improvements for future work.

\section{ACKNOWLEDGEMENTS}

We thank Duncan Forbes and Michele Trenti for sharing with us their expertise in globular cluster formation scenarios. We also thank Michela Mapelli and Christopher Berry for their comments, which improved the manuscript, and our anonymous reviewer, whose suggestions improved both the paper and the science it contains. KK is supported by an NSF Astronomy and Astrophysics Postdoctoral Fellowship under award AST-2001751. PDL and ET are supported through Australian Research Council Future Fellowships FT160100112 and FT150100281, ARC Discovery Project DP180103155, and ARC Centre of Excellence CE170100004. JS is supported by the European Unions Horizon 2020 research and innovation programme under the Marie Skłodowska-Curie Actions grant agreement No. 844629.

\section{DATA AVAILABILITY}

We use publicly available output from the globular cluster simulations of Kremer et al. (2020a), and perform our analysis using publicly available Bayesian inference library bilby (Ashton et al. 2019; Romero-Shaw et al. 2020a).

\section{REFERENCES}

Aasi J. et al., 2015, Class. Quantum Gravity, 32, 074001

Abbott B. P. et al., 2016, Phys. Rev. X, 6, 041015

Abbott B. P. et al., 2017a, Class. Quantum Gravity, 34, 044001

Abbott B. P. et al., 2017b, Phys. Rev. Lett., 119, 161101

Abbott B. P. et al., 2017c, ApJ, 848, L12

Abbott B. P. et al., 2018, Phys. Rev. Lett., 120, 091101

Abbott B. P. et al., 2019, Phys. Rev. X, 9, 031040

Abbott R. et al., 2020a, ApJ, 896, L44

Abbott R. et al., 2020b, Phys. Rev. Lett., 125, 101102

Abbott R. et al., 2020c, Phys. Rev. X, 11, 021053

Abbott R. et al., 2020d, ApJ, 913, L7

Acernese F. et al., 2015, Class. Quantum Gravity, 32, 024001

Antonini F., Gieles M., 2020, Phys. Rev. D, 102, 123016

Antonini F., Toonen S., Hamers A. S., 2017, ApJ, 841, 77

Ashton G. et al., 2019, ApJS, 241, 27

Askar A., Szkudlarek M., Gondek-Rosińska D., Giersz M., Bulik T., 2017, MNRAS, 464, L36

Bavera S. S. et al., 2021, A\&A, 647, A153

Belczynski K., 2020, ApJ, 905, L15

Bethe H. A., Brown G. E., 1998, ApJ, 506, 780

Campanelli M., Lousto C. O., Zlochower Y., 2006, Phys. Rev. D, D74, 084023

Correnti M., Gennaro M., Kalirai J. S., Brown T. M., Calamida A., 2016, ApJ, 823, 18

de Mink S. E., Mandel I., 2016, MNRAS, 460, 3545

de Mink S. E., Cantiello M., Langer N., Pols O. R., 2010, in Kalogera V., van der Sluys M., eds, AIP Conf. Ser. Vol. 1314, Chemically Homogeneous Evolution in Massive Binaries. Am. Inst. Phys., New York, p. 291

Di Carlo U. N., Giacobbo N., Mapelli M., Pasquato M., Spera M., Wang L., Haardt F., 2019, MNRAS, 487, 2947

El-Badry K., Quataert E., Weisz D. R., Choksi N., Boylan-Kolchin M., 2019, MNRAS, 482, 4528 
Elmegreen B. G., 2010, ApJ, 712, L184

Elmegreen D. M., Elmegreen B. G., 2017, ApJ, 851, L44

Fall S. M., Rees M. J., 1985, ApJ, 298, 18

Fishbach M., Holz D. E., 2017, ApJ, 851, L25

Fishbach M., Holz D. E., Farr B., 2017, ApJ, 840, L24

Forbes D. A., Bridges T., 2010, MNRAS, 404, 1203

Forbes D. A., Pastorello N., Romanowsky A. J., Usher C., Brodie J. P., Strader J., 2015, MNRAS, 452, 1045

Forbes D. A. et al., 2018, Proc. R. Soc. A, 474, 20170616

Fragione G., Kocsis B., 2018, Phys. Rev. Lett., 121, 161103

Fragione G., Kocsis B., 2019, MNRAS, 493, 3920

Fregeau J. M., Rasio F. A., 2007, ApJ, 658, 1047

García-Berro E., Torres S., Althaus L. r. G., Miller Bertolami M. M., 2014, A\&A, 571, A56

Gerosa D., Berti E., 2017, Phys. Rev. D, 95, 124046

Gondán L., Kocsis B., 2019, ApJ, 871, 178

Gratton R. G., Fusi Pecci F., Carretta E., Clementini G., Corsi C. E., Lattanzi M., 1997, ApJ, 491, 749

Gröbner M., Ishibashi W., Tiwari S., Haney M., Jetzer P., 2020, A\&A, 638, A119

Hall E. D., Evans M., 2019, Class. Quantum Gravity, 36, 225002

Hansen B. M. S. et al., 2013, Nature, 500, 51

Heger A., Woosley S. E., 2002, ApJ, 567, 532

Hinder I., Vaishnav B., Herrmann F., Shoemaker D., Laguna P., 2008, Phys. Rev. D, D77, 081502

Hoang B.-M., Naoz S., Kocsis B., Rasio F. A., Dosopoulou F., 2018, ApJ, 856,140

Hong J., Vesperini E., Askar A., Giersz M., Szkudlarek M., Bulik T., 2018, MNRAS, 480, 5645

Hurley J. R., Pols O. R., Tout C. A., 2000, MNRAS, 315, 543

Hurley J. R., Tout C. A., Pols O. R., 2002, MNRAS, 329, 897

Ivanova N. et al., 2013, A\&AR, 21, 59

Joshi K. J., Rasio F. A., Zwart S. P., Portegies Zwart S., 2000, ApJ, 540, 969

Kalogera V., 2000, ApJ, 541, 319

Katz H., Ricotti M., 2014, MNRAS, 444, 2377

Kerber L. O. et al., 2019, MNRAS, 484, 5530

Kimball C., Talbot C., Berry C. P. L., Carney M., Zevin M., Thrane E., Kalogera V., 2020a, ApJ, 900, 177

Kimball C. et al., 2020b, preprint (arXiv:2011.05332)

Kimm T., Cen R., Rosdahl J., Yi S. K., 2016, ApJ, 823, 52

Kozai Y., 1962, ApJ, 67, 591

Kremer K. et al., 2020a, ApJS, 247, 48

Kremer K. et al., 2020b, ApJ, 903, 45

Kruckow M. U., Tauris T. M., Langer N., Szécsi D., Marchant P., Podsiadlowski P., 2016, A\&A, 596, A58

Kruijssen J. M. D., 2015, MNRAS, 454, 1658

Lada C. J., Lada E. A., 2003, ARA\&A, 41, 57

Leaman R., VandenBerg D. A., Mendel J. T., 2013, MNRAS, 436, 122

Lidov M. L., 1962, Planet. Space Sci., 9, 719

Lilly S. J., Carollo C. M., Stockton A. N., 2002, preprint (arXiv:astro-ph/0 209243)

Livio M., Soker N., 1988, ApJ, 329, 764

Ma X., Quataert E., Wetzel A., Faucher-Giguère C.-A., Boylan-Kolchin M., 2021, MNRAS, 504, 4062

Madau P., Dickinson M., 2014, ARA\&A, 52, 415

Maggiore M. et al., 2020, J. Cosmol. Astropart. Phys., 2020, 050

Majewski S. R., Patterson R. J., Dinescu D. I., Johnson W. Y., Ostheimer J. C., Kunkel W. E., Palma C., 2000, in Noels A., Magain P., Caro D., Jehin E., Parmentier G., Thoul A. A., eds, Liege International Astrophysical Colloquia Vol. 35, Liege International Astrophysical Colloquia. Institut d'Astrophysique et de Geophysique, Liege, Belgium, p. 619

Morscher M., Pattabiraman B., Rodriguez C., Rasio F. A., Umbreit S., 2015, ApJ, 800, 9

NASA, 2020, NASA Announces New James Webb Space Telescope Target Launch Date. NASA press release. Available at: www.nasa.gov/press-release/nasa-announces-new-james-webb-spa ce-telescope-target-launch-date

Neijssel C. J. et al., 2019, MNRAS, 490, 3740
Pattabiraman B., Umbreit S., Liao W.-k., Choudhary A., Kalogera V., Memik G., Rasio F. A., 2013, ApJS, 204, 15

Peacock M. B., Maccarone T. J., Knigge C., Kundu A., Waters C. Z., Zepf S. E., Zurek D. R., 2010, MNRAS, 402, 803

Peters P. C., 1964, Phys. Rev., 136, B1224

Planck Collaboration XIII,2016, A\&A, 594, A13

Punturo M. et al., 2010, Class. Quant. Grav., 27, 194002

Ramirez-Ruiz E., Trenti M., MacLeod M., Roberts L. F., Lee W. H., SaladinoRosas M. I., 2015, ApJ, 802, L22

Reitze D. et al., 2019, Bull. Am. Astron. Soc., 51, 35

Rodriguez C. L., Antonini F., 2018, ApJ, 863, 7

Rodriguez C. L., Loeb A., 2018, ApJL, 866, L5

Rodriguez C. L., Morscher M., Pattabiraman B., Chatterjee S., Haster C.-J., Rasio F. A., 2015, Phys. rev. lett., 115, 051101

Rodriguez C. L., Chatterjee S., Rasio F. A., 2016a, Phys. Rev. D, 93, 084029

Rodriguez C. L., Zevin M., Pankow C., Kalogera V., Rasio F. A., 2016b, ApJ, 832, L2

Rodriguez C. L., Amaro-Seoane P., Chatterjee S., Rasio F. A., 2018a, Phys. Rev. Lett., 120, 151101

Rodriguez C. L., Amaro-Seoane P., Chatterjee S., Rasio F. A., 2018b, Phys. Rev. Lett., 120, 151101

Rodriguez C. L., Amaro-Seoane P., Chatterjee S., Kremer K., Rasio F. A., Samsing J., Ye C. S., Zevin M., 2018c, Phys. Rev. D, 98, 123005

Romero-Shaw I. M. et al., 2020a, MNRAS, 499, 3295

Romero-Shaw I., Lasky P. D., Thrane E., Calderón Bustillo J., 2020b, ApJ, 903, L5

Safarzadeh M., Berger E., Ng K. K. Y., Chen H.-Y., Vitale S., Whittle C., Scannapieco E., 2019, ApJ, 878, L13

Samsing J., 2018, Phys. Rev. D, D97, 103014

Samsing J., D’Orazio D. J., 2018, MNRAS, 481, 5445

Samsing J., Hotokezaka K., 2020, preprint (arXiv:2006.09744)

Samsing J., MacLeod M., Ramirez-Ruiz E., 2014, ApJ, 784, 71

Samsing J., D’Orazio D. J., Askar A., Giersz M., 2018, preprint (arXiv: 1802.08654)

Sarajedini A. et al., 2007, AJ, 133, 1658

SEDS Messier Database, 2020, Milky Way Globular Clusters. Available at: https://www.messier.seds.org/xtra/supp/mw_gc.html

Shapiro K. L., Genzel R., F'orster Schreiber N. M., 2010, MNRAS, 403, L36

Silsbee K., Tremaine S., 2017, ApJ, 836, 39

Stevenson S., Berry C. P. L., Mandel I., 2017, MNRAS, 471, 2801

Tagawa H., Kocsis B., Saitoh T. R., 2018, Phys. Rev. Lett., 120, 261101

Talbot C., Thrane E., 2017, Phys. Rev. D, 96, 023012

Talbot C., Thrane E., 2018, ApJ, 856, 173

Tamura N., Sharples R. M., Arimoto N., Onodera M., Ohta K., Yamada Y., 2006, MNRAS, 373, 588

Trenti M., Padoan P., Jimenez R., 2015, ApJ, 808, L35

van den Heuvel E. P. J., Portegies Zwart S. F., de Mink S. E., 2017, MNRAS, 471,4256

VandenBerg D. A., Brogaard K., Leaman R., Casagrand e L., 2013, ApJ, 775, 134

Vitale S., Evans M., 2017, Phys. Rev. D, 95, 064052

Vitale S., Whittle C., 2018, Phys. Rev. D, 98, 024029

Vitale S., Farr W. M., Ng K. K. Y., Rodriguez C. L., 2019, ApJ, 886, L1

Wang L. et al., 2016, MNRAS, 458, 1450

Zevin M., Samsing J., Rodriguez C., Haster C.-J., Ramirez-Ruiz E., 2019, ApJ, 871, 91

Zevin M. et al., 2021, ApJ, 910, 152

Zhao W., Wen L., 2018, Phys. Rev. D, 97, 064031

\section{APPENDIX: POSTERIOR PROBABILITY DISTRIBUTIONS FOR POPULATION PARAMETERS}

In this section, we present posterior probability distributions for our five population parameters in cosmic time, recovered using the merger-time distributions of $1 \mathrm{G}$ massive few-body mergers. Posterior distributions are plotted in grey, with the injected values indicated 


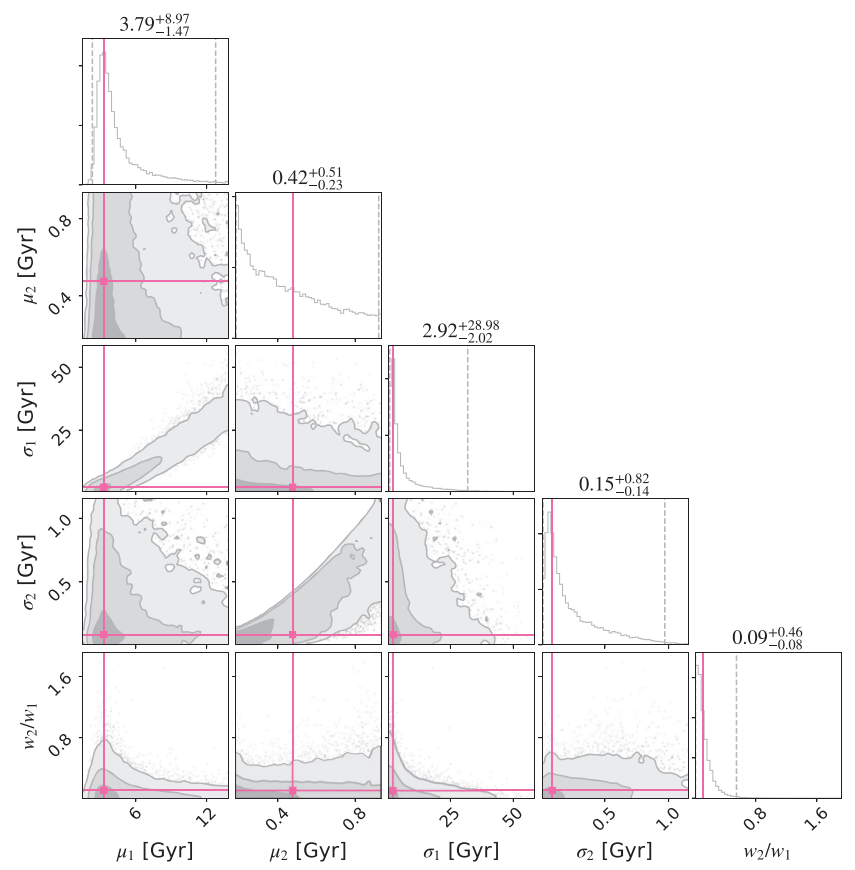

(a)

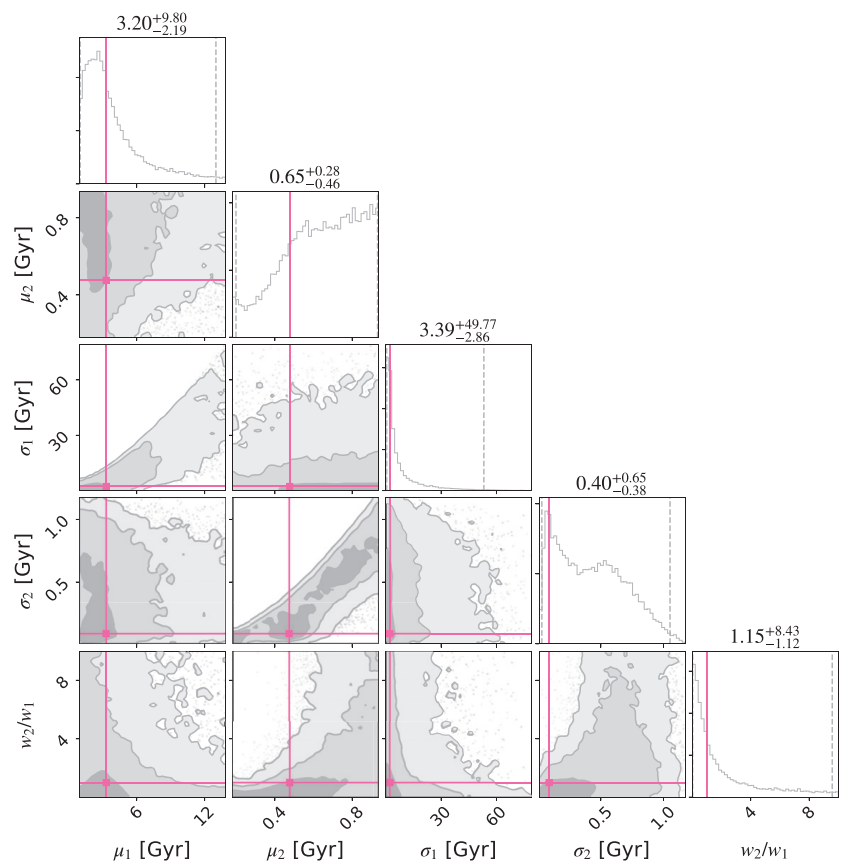

(b)

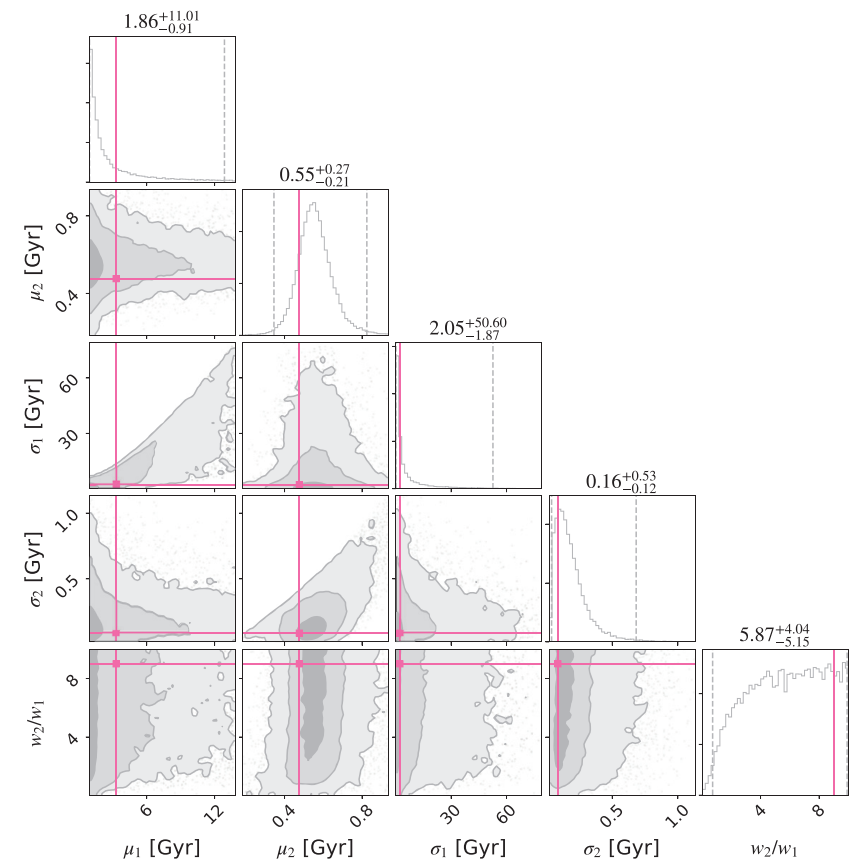

(c)

Figure A1. Posterior probability distributions on our five population parameters after $1 \mathrm{~d}$ of simulated third-generation detector observations of massive few-body mergers from globular clusters ( 25 events). Results are shown for three variations on our two-component Gaussian mixture model: (a) $w_{2} / w_{1}=1 / 9$, (b) $w_{2} / w_{1}=1$, and (c) $w_{2} / w_{1}=9$.

with the pink lines, and the shading gradients on the two-dimensional posteriors indicate levels of $1 \sigma, 2 \sigma$ and $3 \sigma$ credibility, while the grey-dashed error bars around the median recovered values show 99 per cent confidence intervals.

\section{A1 Results after $1 \mathrm{~d}$ of observing}

In Fig. A1, we present the one- and two-dimensional posterior probability distributions over each population parameter resolved after $1 \mathrm{~d}$ of observations with third-generation detectors. 


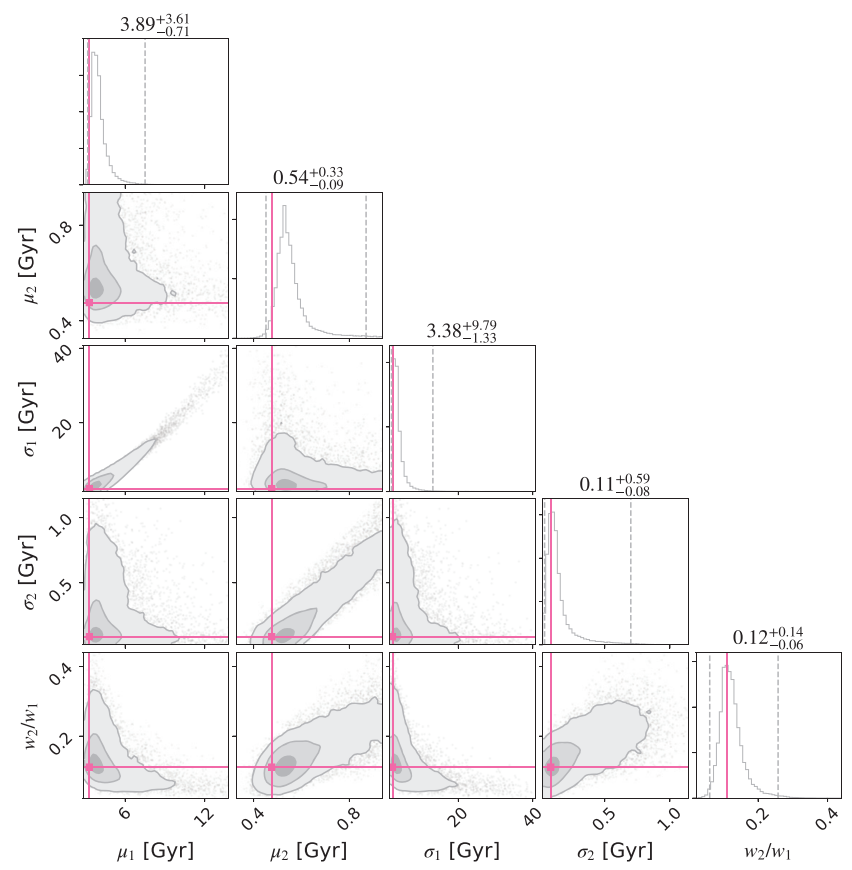

(a)

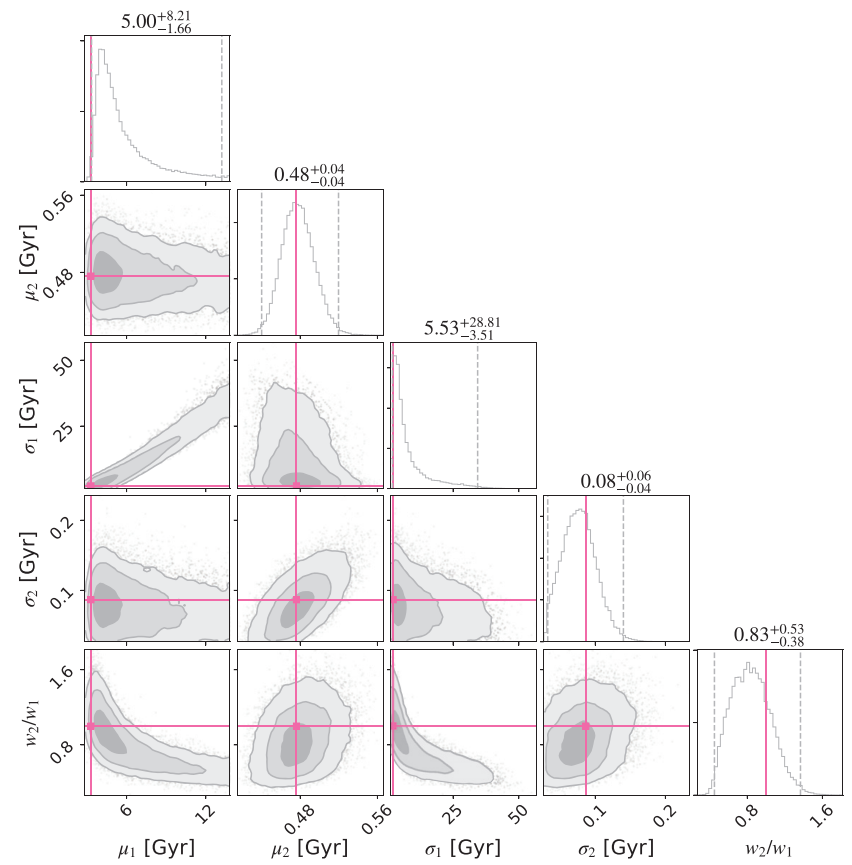

(b)

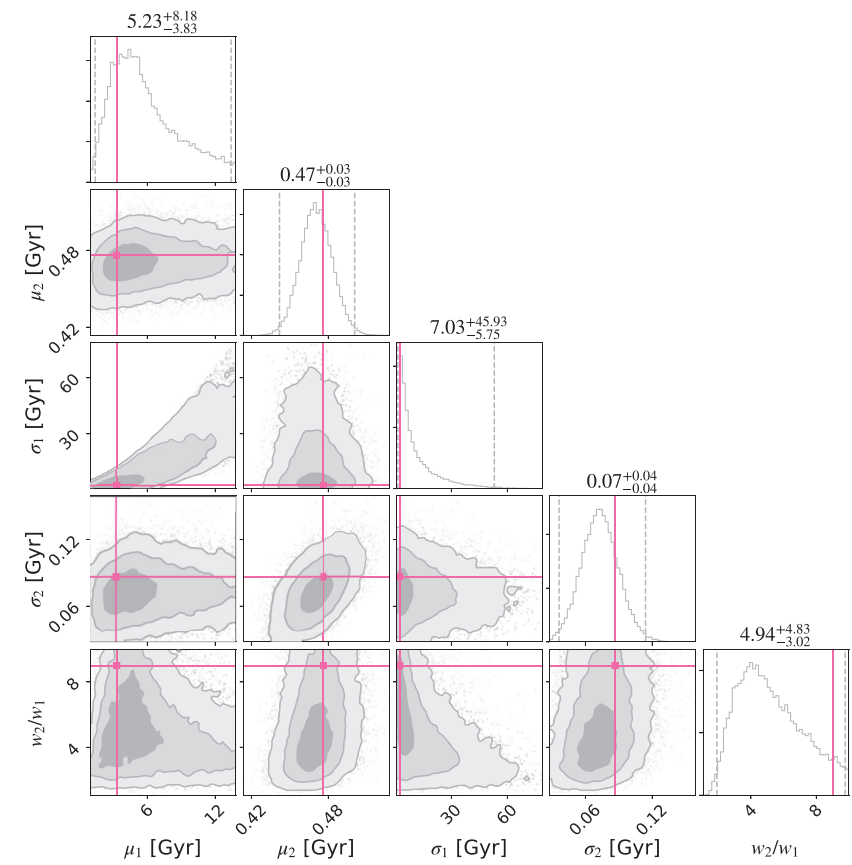

(c)

Figure A2. Posterior probability distributions on our five population parameters after one month of simulated third-generation detector observations of massive few-body mergers from globular clusters (500 events). Results are shown for three variations on our two-component Gaussian mixture model: (a) $w_{2} / w_{1}=1 / 9$, (b) $w_{2} / w_{1}=1$, and (c) $w_{2} / w_{1}=9$.

\section{A2 Results after one month of observing}

In Fig. A2, we present the one- and two-dimensional posterior probability distributions over each population parameter resolved after $1 \mathrm{~d}$ of observations with third-generation detectors.

\section{A3 Results after 1 yr of observing}

In Fig. A3, we present the one- and two-dimensional posterior probability distributions over each population parameter resolved after $1 \mathrm{~d}$ of observations with third-generation detectors. 


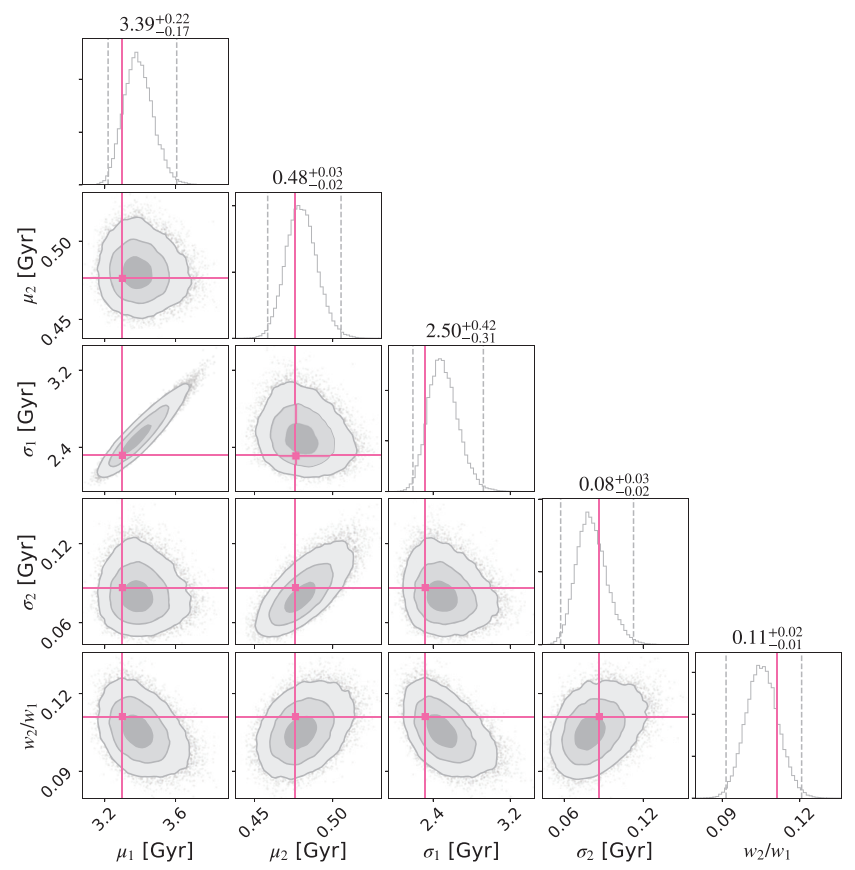

(a)

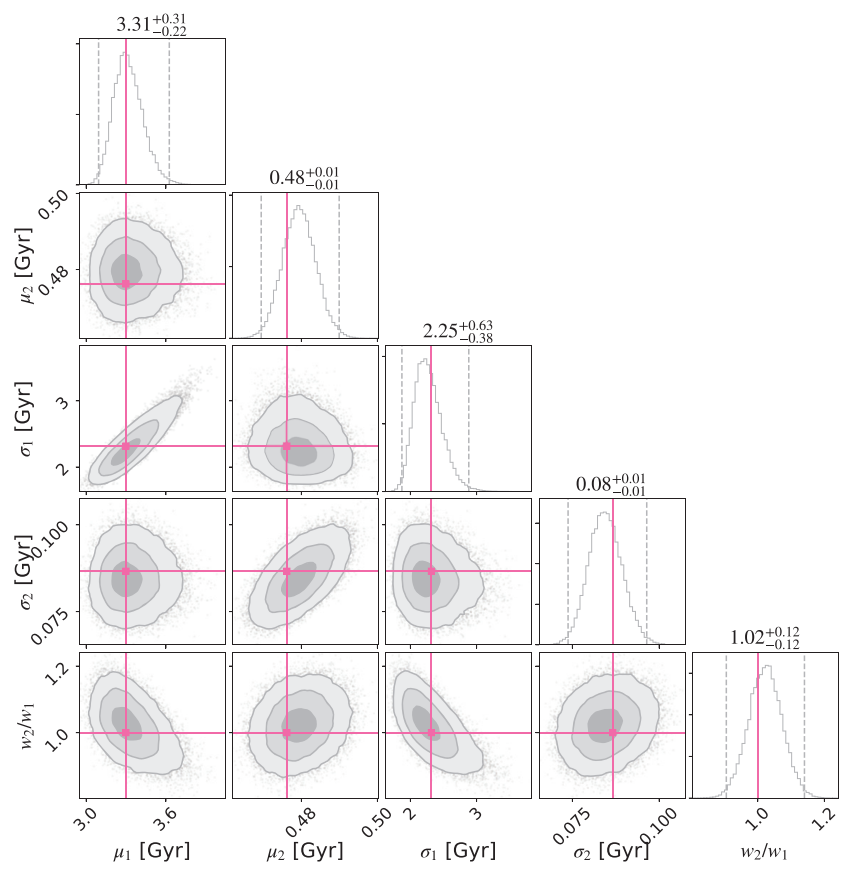

(b)

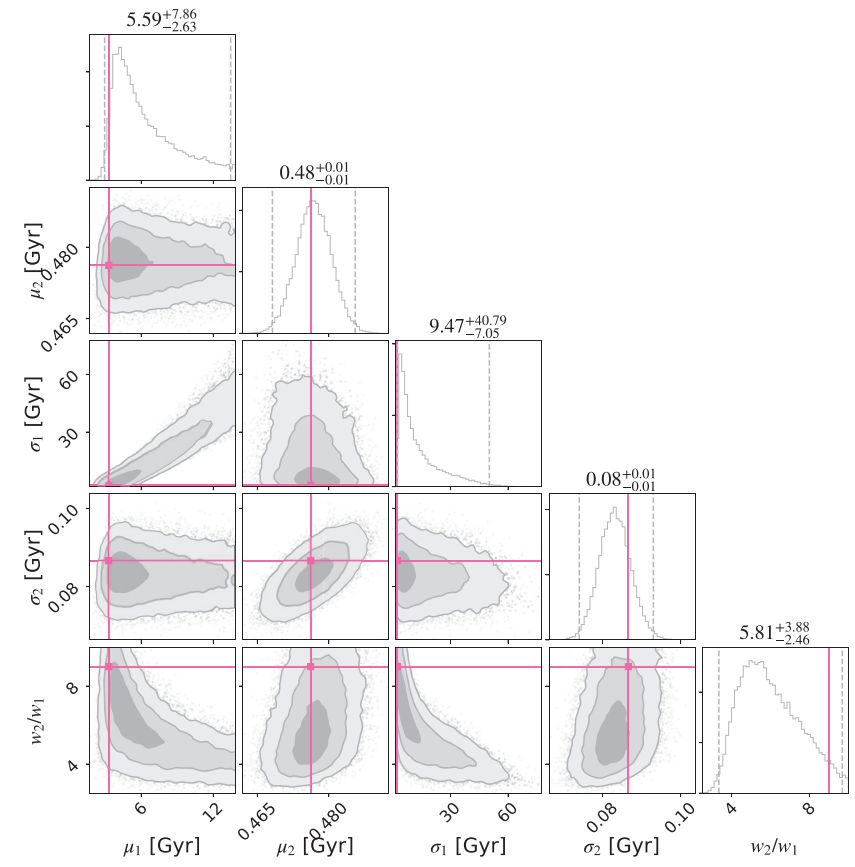

(c)

Figure A3. Posterior probability distributions on our five population parameters after $1 \mathrm{yr}$ of simulated third-generation detector observations of massive few-body mergers from globular clusters (5000 events). Results are shown for three variations on our two-component Gaussian mixture model: (a) $w_{2} / w_{1}=1 / 9$, (b) $w_{2} / w_{1}=1$, and (c) $w_{2} / w_{1}=9$.

This paper has been typeset from a $\mathrm{T}_{\mathrm{E}} \mathrm{X} / \mathrm{LT} \mathrm{E} \mathrm{X}$ file prepared by the author. 